\title{
Indy mutants: live long and prosper
}

\author{
Stewart Frankel ${ }^{1}$ and Blanka Rogina ${ }^{2 *}$ \\ Department of Biology, University of Hartford, West Hartford, CT, USA \\ ${ }^{2}$ Department of Genetics and Developmental biology, School of Medicine, University of Connecticut Health Center, Farmington, CT, USA
}

\section{Edited by:}

Gabrielle L. Boulianne, The Hospital

for Sick Children, Canada

\section{Reviewed by:}

Robert M. Tanguay, Université Laval, Canada

Yousin Suh, Albert Einstein College of Medicine, USA

\section{*Correspondence.}

Blanka Rogina, Department of Genetics and Developmental Biology, School of Medicine, University of Connecticut Health Center, 263 Farmington Avenue, Farmington, CT 06030-6403, USA.

e-mail: rogina@neuron.uchc.edu
Indy encodes the fly homolog of a mammalian transporter of di and tricarboxylate components of the Krebs cycle. Reduced expression of fly Indy or two of the C. elegans Indy homologs leads to an increase in life span. Fly and worm tissues that play key roles in intermediary metabolism are also the places where Indy genes are expressed. One of the mouse homologs of Indy ( $m / n d y)$ is mainly expressed in the liver. It has been hypothesized that decreased INDY activity creates a state similar to caloric restriction (CR). This hypothesis is supported by the physiological similarities between Indy mutant flies on high calorie food and control flies on CR, such as increased physical activity and decreases in weight, egg production, triglyceride levels, starvation resistance, and insulin signaling. In addition, Indy mutant flies undergo changes in mitochondrial biogenesis also observed in $\mathrm{CR}$ animals. Recent findings with $\mathrm{m} / \mathrm{ndy}$ knockout mice support and extend the findings from flies. $m / n d y^{-1-}$ mice display an increase in hepatic mitochondrial biogenesis, lipid oxidation, and decreased hepatic lipogenesis. When $\mathrm{m} / n d y^{-/}$mice are fed high calorie food they are protected from adiposity and insulin resistance. These findings point to INDY as a potential drug target for the treatment of metabolic syndrome, type 2 diabetes, and obesity.

Keywords: Indy, caloric restriction

\section{INTRODUCTION}

Single gene mutations that extend life span are important tools for discovering pathways underlying aging. Of particular interest have been mutations in genes that are conserved across diverse species, since they suggest common mechanisms for aging regulation. For instance, mutations in insulin signaling genes affect the life span of yeast, worms, fruit flies, and mice (Friedman and Johnson, 1988; Kenyon et al., 1993; Morris et al., 1996; Kimura et al., 1997; Clancy et al., 2001; Fabrizio et al., 2001; Tatar et al., 2001; Taguchi et al., 2007).

Mutations in the Indy (I'm Not Dead Yet) gene dramatically extend the life span of the fruit fly, Drosophila melanogaster (Rogina et al., 2000). Indy encodes the fly homolog of a mammalian di and tricarboxylate transporter involved in regulating plasma and liver levels of citrate and other Krebs cycle intermediates (Markovich and Murer, 2004; Pajor, 2006).

\section{INDY IS PART OF THE SLC13 FAMILY OF TRANSPORTERS}

INDY is part of the SLC13 protein family, consisting of $\mathrm{Na}$ carboxylate and $\mathrm{Na}$-sulfate cotransporters in vertebrates, invertebrates, plants, and bacteria (Pajor, 2006). While INDY mediates the exchange of citrate, succinate, and several dicarboxylates across the plasma membrane, all other closely related transporters mediate unidirectional cotransport of di or tricarboxylates with sodium. There are three such Na-carboxylate cotransporters in mammals ( $\mathrm{NaDC} 1, \mathrm{NaDC} 3$, and $\mathrm{NaCT}$ ) and at least three in C. elegans (ceNAC-1, ceNAC-2, and ceNAC-3). The cotransporters in worms and mammals differ by their specificity for di and tri carboxylates, with at least one form that has a higher affinity and/or transport capacity for citrate while the other forms have higher affinity and/or transport capacity for succinate. Fly INDY has 34\% amino acid identity with human liver and renal Na-carboxylate transporters.

\section{PHYSIOLOGICAL FUNCTION OF INDY}

INDY mediates $\mathrm{Na}^{+}, \mathrm{K}^{+}$, and $\mathrm{Cl}^{-}$independent high affinity uptake of citrate, succinate, alpha-ketoglutarate, glutarate, and fumarate across the plasma membrane when expressed in frog oocytes, with the highest rate of uptake for citrate (Knauf et al., 2002). While transport of succinate is $\mathrm{pH}$ independent, uptake of citrate is moderately $\mathrm{pH}$ dependent. Na-independence, electroneutrality, and citrate preference were confirmed by expression in a mammalian cell line (Inoue et al., 2002a,b). Additional studies have shown that INDY mediates bidirectional exchange of dicarboxylate and tricarboxylate Krebs cycle intermediates (Knauf et al., 2006).

\section{INDY MUTATIONS AND LONGEVITY}

The original report of life span extension by Indy heterozygotes used five independent $\mathrm{P}$-element alleles and had as controls precise excisions of the P-elements from two alleles as well as lines derived from the same mutagenesis but lacking any P-elements in the Indy region (Rogina et al., 2000). Indy expression is decreased to varying extents in different P-element insertion alleles (Toivonen et al., 2007; Wang et al., 2009). The level of the INDY protein was shown to be dramatically decreased in flies homozygous for the Indy ${ }^{206}$ allele (Knauf et al., 2002). All alleles had life span extension of $80-100 \%$ when assayed as heterozygotes after crossing to the same wild type background. Indy heterozygotes extend life span 15$80 \%$ when crossed into three additional genetic backgrounds, $H k$, $S h$, and Luckinbill long-lived lines (unpublished data). One of the original alleles was backcrossed into two different control genetic 
backgrounds, $y w$ and $w^{1118}$, in a subsequent study (Wang et al., 2009). The former background showed life span extension in both sexes but not the latter. Another group observed longevity extension in males but not in females with one of the original alleles using the original background and after backcrossing this allele into the $w^{1118}$ genetic background. However, they were unable to obtain life span extension for a second allele in the original background or backcrossed into $w^{1118}$. Both alleles failed to give extension when backcrossed into a different control background, white Dahomey. Treatment for Wolbachia decreased but did not eliminate Indy-induced longevity increases in male flies (Toivonen et al., 2007). The latest study (Wang et al., 2009) offered to reconcile the different results by proposing that the lack of Indymediated life span extension in the Toivonen study may be due to the use of food low in particular nutrients, which is consistent with results linking INDY to caloric restriction (CR; see below). However, these studies highlight the importance of genetic background and variations in experimental conditions such as food preparation. Genetic background also affects the extent to which CR can increase the life span of flies (Bhandari et al., 2007).

RNAi-mediated knockdown of the NAC-2 cotransporter in worms, which has citrate as the preferred substrate, leads to a $20 \%$ increase in life span, decreased body size, and decreased body fat content (Fei et al., 2003, 2004). Knockdown of one of the NAC-3 cotransporters, which prefers succinate, leads to a $15 \%$ increase in life span but no obvious metabolic changes; the other succinate cotransporter has no life span effect. These results could not be replicated in the study by Toivonen et al. (2007).

\section{LOCATION, LOCATION, LOCATION}

Fly INDY is most highly expressed in the gut, fat bodies, and oenocytes. The citrate transporter closest to INDY in the worm is expressed in the intestinal tract (Knauf et al., 2002; Fei et al., 2003). The mouse homolog of INDY is predominantly expressed in liver. In worms and flies the INDY-like citrate transporters are expressed in tissues that function like the liver in mammals, which in invertebrates overlaps with sites of nutrient absorption and fat storage.

\section{INDY AND CALORIC RESTRICTION}

The expression patterns, transport properties, and life span effects of INDY suggest the hypothesis that reduced levels of INDY can induce a condition similar to CR. CR has been shown to increase life span and delay the onset of age-related symptoms across a broad spectrum of organisms, from yeast to flies to mammals (McCay et al., 1935; Weindruch and Walford, 1988). In order to explore if the effect of CR on normal flies is similar to the effect of the Indy mutation, life spans were determined for control, Indy homozygous, and Indy heterozygous flies kept on low $(0.5 \mathrm{~N})$, standard $(1.0 \mathrm{~N})$, or high calorie food (1.5 N; Wang et al., 2009). Life span effects were closely correlated with INDY expression levels. Indy heterozygotes had extended life spans on high and normal calories compared to controls on the same food, but heterozygotes and controls had similar life spans on low calories. Indy homozygotes had extended life span on high calories when compared to controls on the same food, had life spans similar to controls on normal calories, and had lower life spans than controls on low calorie food. When control flies are calorically restricted they have decreased levels of Indy message, providing additional support for the hypothesis (Wang et al., 2009). Levels of mIndy expression were reduced in livers of wild type mice after starvation (Birkenfeld et al., 2011). It will be of interest to further explore how CR modulates Indy mRNA levels.

Several physiological effects also support the parallels between reduced INDY expression and CR. Control flies on CR food and Indy heterozygotes on high calorie food both have higher physical activity, reduced body fat content, decreased body weight, and decreased starvation resistance when compared to controls on high calorie food. Similarly, decreased levels of body fat and size were found in worms with RNAi-induced reductions in INDY expression. How can Indy mutations induce a state similar to CR? One contributing factor could be the insulin signaling pathway, since it is one of the nutrient-sensing pathways. Under standard nutrient conditions insulin signaling is up-regulated and the transcription factor FoxO is phosphorylated, resulting in a cytoplasmic localization. When insulin signaling is downregulated, as in $\mathrm{CR}$, unphosphorylated FoxO enters the nucleus and affects transcription. Two pieces of evidence connect Indy to the insulin signaling pathway. First, the levels of the Drosophila insulin-like peptides (Dilps) dilp2, dilp3, and dilp5 are decreased in CR control flies and Indy mutant flies on a high calorie diet. Second, both Indy mutant flies on a high calorie diet and control flies on CR have increased nuclear localization of FoxO in the fat body (Wang et al., 2009). Additional studies will be necessary to confirm a role for the insulin signaling pathway in INDY-mediated longevity regulation. Such caution is warranted given the complex and controversial relationship between the insulin signaling and CR pathways in worms and flies (Houthoofd et al., 2003; Greer and Brunet, 2009; Partridge et al., 2011; Tatar, 2011).

\section{MITOCHONDRIAL EFFECTS OF INDY MUTATIONS}

One of the initial puzzles of the Indy longevity effects was that the beneficial effect on longevity is not accompanied by significant trade-offs under standard laboratory conditions. Indy mutant flies live longer and are healthier than their genetic controls: they produce more eggs, are more active and have the same metabolic rate, and flight velocity (Marden et al., 2003). However, on lower calorie food Indy mutants were found to have a drastic reduction in fertility (Marden et al., 2003). One approach for determining the mechanism of Indy's effect on longevity is to look for gene regulatory changes (Neretti et al., 2009). Such a study found a decrease in components of the electron transport chain (ETC) of Indy heterozygotes compared to controls (Neretti et al., 2009). The status of the mitochondria in mutant flies was then directly assessed. The activity of ETC complexes I and III was decreased, there was decreased $\mathrm{H}_{2} \mathrm{O}_{2}$ production, decreased oxidative damage with age, unchanged ATP production, and increased density of mitochondria per cell. The transcription factor regulating mitochondrial biogenesis, PCG-1-alpha, was also elevated in Indy flies (Neretti et al., 2009). The findings suggest that decreased flux of electrons through the ETC causes an up-regulation of mitochondria. Neretti et al. (2009) speculate that the combination of decreased flux and increased mitochondrial density leads to a net decrease in reactive oxygen species with maintenance of cellular ATP levels, which could have a positive impact upon longevity. 


\section{INDY KNOCKOUT IN THE MOUSE}

More details about the metabolic role of INDY can be obtained in a mammalian system. The mouse gene with highest similarity to Drosophila Indy, called mIndy (mINDY; SLC13A5) is most abundantly expressed in liver. When expressed in mammalian liver cells mINDY mediates high affinity high capacity plasma membrane transport of citrate and moderate capacity transport of succinate (Birkenfeld et al., 2011; Shulman and Helfand, 2011). The function of mINDY was further investigated in knockout mice. Homozygous mIndy ${ }^{-1}$ mice had reduced uptake of citrate from the blood into the liver, but not other organs, and increased circulating levels of citrate with no change in succinate levels. As was shown for worms, the $m I n d y^{-/}$mice had decreased body weight compared to controls when fed normal food, a trend that became more pronounced with age. This correlated with increases in oxygen consumption, carbon dioxide generation, and resting energy expenditure. The livers of $m I n d y^{-/-}$mice had an increased density of mitochondria and increased expression of PCG-1alpha. Microarray analysis indicated that many of the hepatic gene expression changes associated with CR in control mice were also observed with normally fed $m I n d y^{-/}$mice. This included one result different from the findings in flies, increased expression of ETC components. However, the fly microarray data were obtained from heads and whole thoraces, which include muscle and a portion of the intestinal tract, whereas the mouse microarrays were only performed on liver.

Several findings showed that the loss of mINDY causes metabolic shifts when mice were fed a high fat diet. When $m I n d y^{-/-}$ mice on high fat are compared to controls on the same diet, there is a marked decrease in body weight, an almost $50 \%$ decrease in body fat, and increased lean body mass. Hepatic triglyceride content was reduced by $20 \%$ in the livers of $m I n d y^{-/}$mice fed high fat, accompanied by a marked reduction of visible lipid deposits. A plasma marker of lipid oxidation in the liver was increased $62 \%$. Lipid oxidation can be directly measured in primary hepatocytes isolated from $m I n d y^{-1-}$ mice fed high fat, and was shown to be increased compared to control hepatocytes. The $m I n d y^{-/-}$primary hepatocytes also showed a $90 \%$ reduction in the incorporation of citrate into fatty acids and sterols. The results suggest that the knockout mice fed a high fat diet have reduced liver fat synthesis and significant up-regulation of lipid oxidation. Decreased hepatic ATP production in $m I n d y^{-/}$mice resulted in AMP-activated protein kinase (AMPK) activation. AMPK is activated by a decreased ATP/ADP ratio and promotes fat oxidation, insulin sensitivity, and increased levels of PGC-1-apha. Similar to Indy mutant flies, $m I n d y^{-/-}$mice have increased hepatic mitochondrial biogenesis. The data for $m I n d y^{-/}$mice point to changes in metabolism that protect from adiposity and insulin resistance when kept on a high calorie diet (Birkenfeld et al., 2011).

Metabolic syndrome is a state characterized by increased blood lipid levels, body fat, blood sugar, insulin resistance, and blood pressure. It creates an increased risk of cardiovascular disease, and can be a precursor to pre-diabetes. Mice fed a high fat diet can be used to model metabolic syndrome. $m I n d y^{-1-}$ mice fed a high fat diet appear to be protected from metabolic syndrome. They showed decreased plasma glucose and insulin during an insulin tolerance test. Basal glucose and insulin were also reduced.
Hyperinsulinemic-euglycemic clamps (HE) confirmed that high fat fed $m I n d y^{-/}$mice have an increased insulin sensitivity compared to controls. Endogenous hepatic glucose production was reduced $50 \%$ when blood glucose levels were raised in $m I n d y^{-/-}$ mice, indicating that the liver was one of the target organs with increased insulin sensitivity. It has been hypothesized that ectopic accumulation of diacylglycerol (DAG) mediates the insulin resistance associated with high fat diet by activation of protein kinase Cs (nPKCs; Shulman, 2000; Erion and Shulman, 2010). Interestingly, mIndy ${ }^{-/}$mice fed a high fat diet have $40 \%$ decreased levels of hepatic DAG and consistent with the DAG hypothesis, are protected against insulin resistance caused by high fat diet. The incidence of metabolic syndrome in humans greatly increases with age. Control mice undergo changes in fat mass, body weight, and insulin sensitivity with age, but these changes did not occur in $m I n d y^{-/}$mice with increasing age. In particular, hepatic insulin sensitivity with age was maintained in $m I n d y^{-/-}$mice but not the controls.

Mitochondrial citrate is involved in the production of ATP, whereas cytosolic citrate is involved in the synthesis of fatty acid and inhibition of glycolysis via allosteric control phosphofructokinase- 1 . The citrate transporter on the inner mitochondrial membrane promotes citrate efflux into the cytoplasm. Once in the cytoplasm cytoplasmic citrate can be converted into acetate and oxaloacetate by ATP-citrate lyase, where the acetate can be used for fatty acid synthesis while the oxaloacetate can be converted into malate. Malate can either reenter the mitochondrion via the malate transporter, promoting further efflux of citrate into the cytoplasm, or it can be converted into pyruvate. Experiments in mIndy knockout mice indicate that citrate may be central to regulating energy flux in the liver and perhaps the organism, not malate. If there is excess ATP production in the cell, movement of citrate from the mitochondrion into the cytoplasm provides a way of down-regulating the TCA cycle, inhibiting glycolysis, and promoting energy storage. Plasma membrane citrate transporters provide another means of increasing cytoplasmic citrate levels. The studies in mice and flies show that a decreased level of INDY transporters in the liver or fat bodies leads to higher energy production and lower energy storage. It can be inferred that cytoplasmic citrate levels are highly dependent upon uptake from the plasma or extracellular fluid.

\section{INDY MUTATION PROMOTES EXTENDED HEALTH}

Flies, worms, and mice show similar physiological changes when INDY expression is reduced, suggesting an evolutionarily conserved role for INDY in the regulation of metabolism. This stems from INDY-mediated control of citrate, a common factor in fat synthesis, glycolysis, and ATP production in the mitochondrion. Studies on the livers of $m I n d y$ knockout mice indicate that reduced import of citrate from the plasma into the cytoplasm greatly reduces fat synthesis, increases fat oxidation, while stimulating mitochondrial biogenesis.

The results on flies and mice fed a high fat diet were particularly intriguing and important because they highlight a role for INDY in maintaining mammalian health during aging or metabolic disease. Both species were protected from the adverse effects of high fat diets when they had reduced levels of INDY. The studies on mice further suggest protection from metabolic syndrome 
and insulin resistance, whether the causative factors are diet or age. INDY is thus an attractive drug target for the amelioration of metabolic disorders associated with diet or advanced age, separate from any effects on longevity. Part of the attraction is the cellular location and tissue distribution of INDY in mammals, the plasma membrane of the liver. An inhibitory drug would be specific in its tissue effects and the target protein would be accessible to drugs circulating in the blood. Further research should elucidate the

\section{REFERENCES}

Bhandari, P., Jones, M. J., Martin, I., and Grotewiel, M. S. (2007). Dietary restriction alters demographic but not behavioral aging in Drosophila. Aging Cell 6, 631-637.

Birkenfeld, A. L., Lee, H.-Y., GuebreEgziabher, F., Alves, T. C., Jurczak, M. J., Jornayvaz, F. R., Zhang, D., Hsiao, J. J., Martin-Montalvo, A., FischerRosinsky, A., Spranger, J., Pfeiffer, A. F., Jordan, J., Fromm, M. F., König, J., Lieske, S., Carmean, C. M., Frederick, D. W., Weismann, D., Knauf, F., Irusta, P. M., De Cabo, R., Helfand, S. L., Samuel, V. T., and Shulman, G. I. (2011). Deletion of the mammalian INDY homolog mimics aspects of dietary restriction and protects against adiposity and insulin resistance in mice. Cell Metab. 14, 184-195.

Clancy, D. J., Gems, D., Harshman, L. G., Oldham, S. Stocker, H., Hafen, E., Leevers, S. J., and Partridge, L. (2001). Extension of life-span by loss of CHICO, a Drosophila insulin receptor substrate protein. Science 292, 104-106.

Erion, D. M., and Shulman, G. I. (2010). Diacylglycerol-mediated insulin resistance. Nat. Med. 16, 400-402.

Fabrizio, P., Pozza, F., Pletcher, S. D., Gendron, C. M., and Longo, V. D. (2001). Regulation of longevity and stress resistance by Sch9 in yeast. Science 292, 288-290.

Fei, Y. J., Inoue, K., and Ganapathy, V. (2003). Structural and functional characteristics of two sodiumcoupled dicarboxylate transporters (ceNaDC1 and ceNaDC2) from Caenorhabditis elegans and their relevance to life span. J. Biol. Chem. 278, 6136-6144.

Fei, Y. J., Liu, J. C., Inoue, K., Zhuang, L., Miyake, K., Miyauchi, S., and andGanapathy, V. (2004). Relevance of NAC-2, an Na+-coupled citrate transporter, to life span, body size and fat content in Caenorhabditis elegans. Biochem. J. 379, 191-198.

Friedman, D. B., and Johnson, T. (1988). A mutation in the age-1 gene in
Caenorhabditis elegans lengthens life and reduces hermaphrodite fertility. Genetics 118, 75-86.

Greer, E. L., and Brunet, A. (2009). Different dietary restriction regiments extend lifespan by both independent and overlapping genetic pathways in C. elegans. Aging Cell 8, 113-127.

Houthoofd, K., Braeckman, B. P., Johnson, T. E., and Vanfleteren, J. R. (2003). Life extension via dietary restriction is independent of the Ins/IGF-1 signalling pathway in Caenorhabditis elegans. Exp. Gerontol. 38, 947-954.

Inoue, K., Fei, Y. J., Huang, W., Zhuang, L., Chen, Z., and Ganapathy, V. (2002a). Functional identity cation-independent, electroneutral transporter for tricarboxylic acidcycle intermediates. Biochem. J. 367, 313-319.

Inoue, K., Zhuang, L., Maddox, D. M., Smith, S. B., and Ganapathy, V. (2002b). Human sodium-coupled citrate transporter, the orthologue of Drosophila Indy, as a novel target for lithium action. Biochem. J. 374, 21-26.

Kenyon, C., Chang, J., Gensch, E., Rudner, A., and Tabtiang, R. (1993). A C. elegans mutant that lives twice as long as wild type. Nature 366, 461-464.

Kimura, K. D., Tissenbaum, H. A., Liu, Y., and Ruvkun, G. (1997). daf-2, an insulin receptor-like gene that regulates longevity and diapause in Caenorhabditis elegans. Science 277, 942-946.

Knauf, F., B. Rogina, Jiang, Z., Aronson, P. S., and Helfand, S. L. (2002). Functional characterization and immunolocalization of the transporter encoded by the lifeextending gene Indy. Proc. Natl. Acad. Sci. U.S.A. 99, 14315-14319.

Knauf, F., N. Mohebbi, N., Teichert, C., Herold, D., Rogina, B., Helfand, S., Gollasch, M., Luft, F. C., and Aronson, P. S. (2006). The life-extending gene Indy encodes an exchanger for Krebs-cycle intermediates. Biochem. J. 397, 25-29. of Drosophila melanogaster Indy as a

role of INDY in metabolic regulation and could provide valuable information about mechanisms underlying healthy aging and life extension.

\section{ACKNOWLEDGMENTS}

We thank Dr. Stephen L. Helfand for critical reading of the manuscript. This work was supported by grant from the NIH (AG023088 to Blanka Rogina).

Marden, J., Rogina, B., Montooth, K. L. and Helfand, S. L. (2003). Conditional tradeoffs between aging and organismal performance of Indy long-lived mutant flies. Proc. Natl. Acad. Sci. U.S.A. 100, 3369-3373.

Markovich, D., and Murer, H. (2004). The SLC13 gene family of sodiumsulphate/carboxylate cotransportes. Pflugers Arch. 447, 594-602.

McCay, C. M., Crowell, M. F., and Maynard, L. A. (1935). The effect of retarded growth upon the lenght of life span and upon the ultimate body size. J. Nutr. 10, 63-79.

Morris, J. Z., Tissenbaum, H. A., and Ruvkun, G. (1996). A phosphatidylinositol-3-OH kinase family member regulating longevity and diapause in Caenorhabditis elegans. Nature 384, 536-539.

Neretti, N., Wang, P.-Y., Brodsky, A. S., Nyguyen, H. H., White, K. P., Rogina, B., and Helfand, S. L. (2009). Long-lived Indy induces reduced mitochondrial ROS production and oxidative damage. Proc. Natl. Acad. Sci. U.S.A. 106, 2277-2282.

Pajor, A. M. (2006). Molecular properties of the SLC13 family of dicarboxylate and sulfate transporters. Pflugers Arch. 451, 597-605.

Partridge, L., Alic, N., Bjedov, I., and Piper, M. D. W. (2011). Ageing in Drosophila: the role of the insulin/Igf and TOR signaling network. Exp. Gerontol. 46, 376-381.

Rogina, B., Reenan, R. A., Nielsen, S. P. and Helfand, S. L. (2000). Extended life-span conferred by cotransporter gene mutations in Drosophila. Science 290, 2137-2140.

Shulman, G. I. (2000). Cellular mechanisms of insulin resistance. J. Clin. Invest. 106, 171-176.

Shulman, G. I., and Helfand, S. L. (2011). Indy knockdown in mice mimetics elements of dietary restriction. Aging 3, 1.

Taguchi, A., Wartschow, L., and White, M. F. (2007). Brain IRS2 signaling coordinates life span and nutrient homeostasis. Science 317, 469-372.

Tatar, M. (2011). The plate half-full: status of research on the mechanisms of dietary restriction in Drosophila melanogaster. Exp. Gerontol.46, 363368.

Tatar, M., Kopelman, A., Epstein, D., Tu, M. P., Yin, C. M., and Garofalo R. S. (2001). A mutant Drosophila insulin receptor homolog that extends life-span and impairs neuroendocrine function. Science 292, 107-110.

Toivonen, J. M., Walker, G. A., MartinezDiaz, P., Bjedov, I., Driege, Y., Jacobs, H. T., Gems, D., and Partridge, L. (2007). No influence of Indy on lifespan in Drosophila after correction for genetic and cytoplasmic background effects. PLoS Genet. 3, e95. doi:10.1371/journal.pgen.0030095

Wang, P. Y., Neretti, N., Whitaker, R., Hosier, S., Chang, C. Lu, D. Rogina, B., and Helfand, S. L. (2009). Long-lived Indy and calorie restriction interact to extend life span. Proc. Natl. Acad. Sci. U.S.A. 106, 9262-9267.

Weindruch, R., and Walford, R. L. (1988). The Retardation of Aging and Disease by Dietary Restriction. Springfield, IL: C. C. Thomas.

Conflict of Interest Statement: The authors declare that the research was conducted in the absence of any commercial or financial relationships that could be construed as a potential conflict of interest.

Received: 20 October 2011; accepted: 21 January 2012; published online: 17 February 2012.

Citation: Frankel $S$ and Rogina $B$ (2012) Indy mutants: live long and prosper. Front. Gene. 3:13. doi: 10.3389/fgene.2012.00013

This article was submitted to Frontiers in Genetics of Aging, a specialty of Frontiers in Genetics.

Copyright (C) 2012 Frankel and Rogina. This is an open-access article distributed under the terms of the Creative Commons Attribution Non Commercial License, which permits non-commercial use, distribution, and reproduction in other forums, provided the original authors and source are credited. 